\title{
Valoración de la supervisión en doctorados internacionales online por personal director de tesis y estudiantes
}

\author{
Students and Supervisors' Evaluation of Online International Doctoral Supervision
}

\author{
Avaliação da supervisão de doutorados internacionais on-line \\ por orientadores de teses e estudantes
}

\begin{abstract}
Resumen: En este trabajo se presentan los resultados de un estudio sobre los procesos de supervisión online de tesis doctorales en el ámbito de las ciencias de la educación. La supervisión y tutoría remota presentan nuevos problemas en el desarrollo de doctorados de alto nivel con una base internacional de estudiantes dispersa, madura y a tiempo parcial. Estos procesos son analizados a partir de la dinámica de la supervisión y de los sistemas de comunicación y de apoyo utilizados, de los espacios de actividad y las herramientas utilizadas. Se optó por la entrevista semiestructurada con una parte abierta -a modo de entrevista en profundidad- a la que se añadieron preguntas cerradas de ordenar o valorar afirmaciones -con un carácter confirmatorio-. Fueron realizadas 26 entrevistas a personal supervisor y a estudiantes, lo que permitió una mirada desde ambos lados para una mejor comprensión del proceso de supervisión y sus dificultades. Se estableció un sistema de categorización a posteriori, a partir de las notas de las entrevistas tomadas in situ y, posteriormente, las categorías se aplicaron a la transcripción de las entrevistas. Se han identificado elementos clave en los que intervenir para mejorar el proceso. Al tener en cuenta los puntos de vista del personal supervisor como de estudiantes, se pudo constatar cierta coincidencia al identificar dichos elementos, tanto en relación con el estilo de supervisión y apoyo al proceso, como en relación con la dinámica o a las herramientas y aplicaciones susceptibles de mejorar el proceso, aunque ambos colectivos no le atribuyen el mismo valor.
\end{abstract}

Palabras clave: Métodos de supervisión; programas de doctorado; supervisión de doctorado; supervisión online, formación en investigación.

\footnotetext{
${ }^{1}$ Doctor en Filosofía y Ciencias de la Educación. Catedrático de Didáctica y Orgnaización Escolar de la Universitat de les Illes Balears. Investigador Principal del Grup de Tecnologia Educativa. Director del Institut de Recerca i Innovació Edcuativa (UIB). Co-director del Master Interuniversitario en Tecnología Educativa: E-learning y Gestión del Conocimiento (2001-2015), y coordinador del Doctorado en Tecnología Educativa (UIB, URV, U. Lleida y U. Murcia). Director de la Maestría en Educación en Entornos Virtuales de la Universidad Nacional de la Patagonia Austral (Argentina), Robert de Kieffer International Fellowship Award, 2006 de la AECT. Director de Edutec Revista electrónica de Tecnología Educativa. Más infomación en http://gte.uib.es/pape/gte/users/jsalinas
} 
doi: http://dx.doi.org/10.15359/ree.22-1.4

URL: http://www.una.ac.cr/educare

CORREO: educare@una.cr

\begin{abstract}
This research presents the initial results of a study on the processes of online PhD supervision in the Education Sciences field. Remote mentoring and supervision bring new problems to the delivery of high-level doctoral studies with a widely dispersed base of mature, part-time, international students. These processes are analyzed by taking into account the supervision dynamics, the communication and support systems used, the activity space developed, and the tools used. For this exploratory study, we opted for a semi-structured interview; open ended at first, but to which some closed questions were added (especially questions to order or statements to evaluate). Twenty-six supervisors and students were interviewed, affording a view from both sides, so as to better understand the process and its difficulties. A classification system was set up afterwards, using notes taken on the spot in the interviews, and, later on, the categories were applied to the interview transcripts. Key elements have been identified, in which to intervene to improve the process. By taking into account the supervisors' and students' points of view, a certain coincidence could be observed when identifying such elements, both in relation to the style of supervision and support to the process, as well as to the dynamics, or susceptible tools and applications to improve the process; although both groups do not attribute the same value.
\end{abstract}

Keywords: Supervisory methods; doctoral programs; doctoral supervision; online supervision; research training.

Resumo: Este artigo apresenta os resultados de um estudo sobre processos de supervisão on-line em teses doutorais no campo das ciências da educação. $\mathrm{O}$ acompanhamento e orientação à distância apresentam novos problemas no desenvolvimento de doutorados de alto nível com uma base internacional de estudantes dispersa, madura e a tempo parcial. Estes processos são analisados a partir da dinâmica de supervisão e de sistemas de comunicação e apoio, espaços de atividades e técnicas utilizadas. Foi feito através de entrevista semiestruturada com uma parte de perguntas abertas, uma forma de entrevista em profundidade acompanhada por perguntas fechadas sobre organizar ou avaliar afirmações, com caráter confirmativo. Foram realizadas 26 entrevistas com o pessoal de supervisão e estudantes, permitindo uma visão de ambos os lados para uma melhor compreensão do processo de monitoramento e suas dificuldades. Um sistema de categorização foi estabelecido respectivamente, a partir das notas das entrevistas realizadas e posteriormente, das categorias aplicadas na transcrição das entrevistas. Foram identificaram elementos-chave que necessitam intervenção para melhorar o processo. Tendo em conta os pontos de vista do pessoal de supervisão e estudantes, verificou-se alguma coincidências na identificação desses elementos, tanto em relação ao estilo de supervisão e apoio ao processo, como em relação à dinâmica ou técnicas e aplicações suscetíveis em melhorar o processo, embora ambos os grupos não dão o mesmo valor ao tema.

Palavras-chave: Métodos de supervisão; programas de doutorado; supervisão de doutorado; supervisão on-line, formação em investigação.

\title{
Introducción
}

Los avances recientes del aprendizaje en red y los cambios que ha traído el Espacio Europeo de Educación Superior han promovido un incremento de programas de doctorado a distancia y una diversificación en las formas de desarrollar la investigación en estos mismos (European University Association, EUA, 2007). En este contexto de cambio, se dispone de diversos estudios 
sobre el doctorado, sobre sus desafíos en las políticas y también en las prácticas, sobre los cambios en los modos de producción del conocimiento, en la internacionalización del cuerpo de estudiantes, tanto en USA (Golde, 2005); como en Australia (Gilbert, 2004), o la UE (EUA, 2007). Al mismo tiempo, se plantean retos adicionales en relación con la iniciación del estudiantado a distancia en la comunidad académica o a la incorporación de este a los equipos de investigación de forma equivalente a las tradicionales en el campus, en relación con el estudiantado a tiempo parcial o a los doctorados profesionales, entre otros.

El presente trabajo se ocupa del caso particular de los doctorados en Ciencias de la Educación en español en los que participan estudiantes latinoamericanos y que suelen ser programas de responsabilidad total o compartida de universidades españolas. Aunque coexisten distintos formatos de programas de doctorado, se trata de estudiantes que han seguido una fase de formación de dos cursos, seguida de una fase de investigación en la que se genera la tesis. Son doctorados en Ciencias de la Educación cuyo estudiantado se caracteriza por la internacionalización, ser de edad madura, a tiempo parcial. En el caso de estudio, siguen la fase de investigación a distancia y en casi ningún caso dejan totalmente sus obligaciones en la propia institución. Así, en lugar de un doctorado en el que la dedicación es similar al trabajo remunerado para un candidato o candidata a tiempo completo, para el caso de estudiantes a tiempo parcial, por lo general se trata de una actividad que debe comprimir junto con su trabajo.

De esta manera, se sigue una dinámica de supervisión particular, donde quien supervisa e investigadores e investigadoras en formación, al no coincidir habitualmente cara a cara, utilizan sistemas de comunicación online (Erichsen, Bolliger y Halupa (2014). Por otra parte, no suelen utilizar habitualmente recursos de investigación de la institución responsable del programa de doctorado, lo cual requiere un plus de apoyo en relación con los recursos necesarios para investigar, aun cuando a veces utilicen los recursos de la propia institución.

Cuando personal supervisor y estudiantes tienen su origen en culturas diversas, aparecen factores culturales adicionales que deben ser tomados en cuenta en la pedagogía de la supervisión. En un programa de doctorado como el de este estudio, quien supervisa está en posición de influir en el éxito del estudiantado internacional; por lo tanto, debe entender las expectativas de los investigadores e investigadoras en formación respecto a la supervisión y el enfoque de aprendizaje. La noción postcolonial de la "zona de contacto" sirve para reconceptualizar la naturaleza del trabajo en la investigación (Manthunga 2009), desde el momento en que la diferencia cultural se percibe como productiva, más que como un déficit. Participar en la investigación en la zona de contacto intercultural permite al estudiantado investigador aprovechar sinergias, interconexiones y relaciones para generar nuevos conocimientos y formas de dirección de la investigación. 
doi: http://dx.doi.org/10.15359/ree.22-1.4

URL: http://www.una.ac.cr/educare

CORREO: educare@una.cr

El estudio parte de la concepción de supervisión como una práctica pedagógica (Green, 2009; Malfroy, 2005) y de la perspectiva práctica en la educación doctoral de Lee y Bound (2009), y del que involucra a actores, acciones, escenarios, herramientas y artefactos, reglas, roles y relaciones. Aquí nos centramos en la educación doctoral 'como' práctica en contraposición a la educación doctoral 'para' la práctica (Green, 2009; Villardón-Gallego y Yániz, 2013), aspectos ambos de actualidad en el debate sobre el doctorado. El trabajo se centra en la experiencia de investigadores e investigadoras, ya sea en función de supervisión, ya sea en formación, en la negociación de los desafíos de la supervisión online a tiempo parcial. En particular analiza:

- El estilo de supervisión y el apoyo al proceso de un doctorado internacional online.

- La perspectiva del personal supervisor y estudiante de la experiencia vivida en el proceso.

- El tipo de aplicaciones y herramientas de red que son consideradas adecuadas para este tipo de supervisión.

En otras palabras, el foco está en los micro-niveles de la práctica, atiende la dinámica de la supervisión y los sistemas de comunicación y de apoyo utilizados, los espacios de actividad desarrollados y las herramientas utilizadas.

\section{Marco de referencia}

Preparar al estudiantado para elaborary completar una tesis doctoral es un asunto complejo en los programas on-campus y es comprensible que la experiencia en línea de la investigación doctoral funcione de forma diferente, presente mayores dificultades y demande cambios en los roles de supervisión (Price y Money, 2002). Esto afecta fuertemente al funcionamiento de los cursos de postgrado (master y sobre todo doctorado), en cuanto a la culminación con éxito de los trabajos de investigación personal (tesis, etc.). Pero también afecta a los equipos de investigación que albergan dichas investigaciones (mejora en las técnicas y procedimientos de supervisión de trabajos por parte de doctores y doctoras jóvenes, fortalecimiento de la tutoría colaborativa, incorporación de nuevos elementos al grupo, etc.).

Uno de los aspectos más sensibles de los doctorados a distancia es el apoyo y supervisión de los trabajos de investigación. Cuando estos doctorados se apoyan en sistemas de comunicación instantánea a través medios como e-mail, videoconferencia y Virtual Learning Environments (VLEs) aparecen características y elementos de análisis específicos, aunque muchos de los resultados de los estudios sobre la supervisión de trabajos de investigación on-campus como los que se ocupan de la interacción entre estudiantado y personal supervisor, o de las estructuras y procedimientos, del estilo, la competencia, la actitud y el nivel académico, pedagógico e intelectual de quien supervisa son aplicables o al menos adaptables en nuestro caso. Para 
Macauley (2002), la bibliografía sobre doctorados a distancia siempre hace referencia al factor emocional del aislamiento de los candidatos y candidatas, y tiende a centrarse en la ausencia de cultura de investigación de estos grupos, la falta de masa crítica y la naturaleza problemática del apoyo a la supervisión. Entre las investigaciones sobre la supervisión han sido identificados como indicadores significativos de la finalización del estudiantado candidato (Sinclair, 2004): el grado de la asistencia (a tiempo parcial o completo) (Watts, 2010) y la frecuencia y relevancia de la interacción (Lee 2008; Seagram, Gould y Pyke 1998). Otros indicadores identificados son la disponibilidad de financiación para la investigación, la edad, la excelencia de un expediente previo, la disciplina (ciencias o humanidades) (Seagram et al. 1998; Sinclair 2004), el género, la idoneidad, el tema de investigación, el ambiente intelectual del departamento, y el acceso a equipamiento adecuado y a los ordenadores.

En cualquier caso, la supervisión de doctorado, y más si esta se desarrolla a distancia, presenta todavía muchos aspectos por conocer. Al mismo tiempo, quedan pendientes debates y estudios sobre el mismo concepto de supervisión (Green, 2009; Lee y Boud, 2009). Para Edwards (2002), existen cuatro problemas importantes en la experiencia de posgrado: cuando se tienen propósitos encontrados con las personas supervisoras, cuando se encuentra pocas estructuras de apoyo, el aislamiento y la confusión sobre los recursos. El asilamiento es, en efecto, un aspecto recurrente en la bibliografía sobre el tema (Hartikainen, Suhonen y Sutinen, 2006; Macauley, 2002).

Otro de los aspectos más estudiado lo constituye, sin duda, la calidad de las prácticas de supervisión que tienen un efecto demostrable en los resultados de postgrado. La supervisión efectiva de investigadores e investigadoras en formación es un complejo proceso multifactorial que abarca cuestiones como la relación del estudiantado y personal supervisor en todos los niveles, el apoyo de la infraestructura disponible, las políticas institucionales y gubernamentales, estructuras y procedimientos (Latona y Browne 2001; Sinclair, 2004). Conocer cómo surgen estos factores en los programas de doctorado online ayuda a mejorar el proceso de supervisión. De ellos, la interacción personal supervisor-estudiantado es un factor clave. El estilo, la competencia, la actitud y el nivel académico e intelectual del personal supervisor son los elementos importantes en esta interacción. Junto a ello, se requiere un conjunto de destrezas académicas, interpersonales y pedagógicas para una interacción exitosa (Latona y Browne 2001; Lee 2008; Orellana, Darder, Perez y Salinas, 2016; Seagram et al. 1998; Vilkinas 2008; Woolderink, Putnik, van der Boom y Klabbers, 2015). Hartikainen et al. (2006) y Macauley (2002) remarcan la importancia de la interacción y del estilo y capacidades del personal que supervisa cuando la supervisión se lleva a cabo online.

Se espera que la experiencia de investigación de doctorado off-campus funcione de manera diferente en comparación con un campus a tiempo completo. Hay aspectos en los que coinciden, pero en otros se agudizan las debilidades detectadas en anteriores estudios 
doi: http://dx.doi.org/10.15359/ree.22-1.4

URL: http://www.una.ac.cr/educare

CORREO: educare@una.cr

(Hartikainen et at., 2006; Wikeley y Muschamp, 2004). Se identifican, como ventajas, la flexibilidad, comodidad, y la eliminación de las barreras de viajes y de distancia, y como desventajas, los fallos tecnológicos, la falta de experiencia con la tecnología informática, la falta de contacto humano, las limitadas oportunidades para ver la comunicación no verbal, y la limitada vinculación entre quien supervisa y estudiante.

Atendiendo a la supervisión de este tipo de estudiantes, Evans (2010) indica que, si establecer una buena relación de trabajo con uno o una estudiante de doctorado es esencial, hacerlo con estudiantes a tiempo parcial es clave. En esta línea, para Watts (2010), una práctica efectiva de supervisión para estudiantes a tiempo parcial puede clasificarse en tres categorías: comunicación, planificación y empatía. Para Erichsen et al. (2014), tiene especial relevancia desde la perspectiva de la percepción de cada estudiante, el apoyo, la comunicación. Esto no quiere decir que la tecnología u otros elementos ya documentados por Combe (2005) no sean importantes, sino que en su lugar se argumenta que la supervisión y los métodos de calidad estarían incluidos en estos. En otras palabras, existen algunas evidencias que sugieren que una buena estructura del programa requiere tener un sistema de gestión de aprendizaje (LMS). Del mismo modo, hay evidencias que sugieren que la calidad de la supervisión supone conocer las mejores prácticas en el uso de e-learning y en la capacidad para guiar los doctorandos en el uso de la tecnología (Strang, 2009). En este contexto, al abordar el tema desde el manejo del proceso de supervisión off-campus, con estudiantes a tiempo parcial aparecen, como elementos de análisis y sobre los cuales intervenir para la mejora, los siguientes:

- El apoyo proporcionado y el estilo de supervisión (Watts, 2010).

- La dinámica de comunicación tanto desde la perspectiva de intercambio académico como de refuerzo emocional (Erichsen et al., 2014; Ives y Rowley, 2005; Lee, 2008).

- La organización del espacio de actividad alrededor de las herramientas adecuadas para el desempeño de las funciones a potenciar en el proceso de supervisión (Winston \& Fields, 2003).

Para avanzar en esta vía y averiguar el estado actual de estos aspectos, resulta importante examinar e identificar los principales elementos que contribuyen a la vida intelectual de investigadores e investigadoras en formación en un entorno de campus tradicional y que se han de trasladar o adaptar para que funcionen en un ambiente virtual. Unwin (2007) sugiere algunos de estos elementos, aunque advierte que algunos son más fáciles de incorporar en un entorno virtual que otros, tales como la inducción, el acceso a los recursos bibliográficos, participación en seminarios y reuniones académicas, el proceso formal de revisión anual, 
reuniones de supervisión con pares, discusiones individuales con el personal supervisor sobre el desarrollo del proyecto de investigación, comentarios de quien supervisa en el trabajo escrito, y networking regular y discusiones con pares de doctorado en el departamento y en otros lugares.

En paralelo, es necesario activar tanto espacios de actividad virtuales para la comunicación personal supervisor/estudiantado supervisado y entre pares, como metodologías nuevas que contribuyan por una parte al desarrollo de competencias y roles en el ejercicio profesional, que en nuestro caso supone competencias de investigación y de enseñanza, y, por otra, desarrollar habilidades de aprendizaje relevantes a lo largo de sus estudios de postgrado y más allá (Lessing y Shulze 2003; Price y Money, 2002). Es verdad que la evolución en el campo y la mayor variedad de entornos e-learning que aportan una mayor riqueza de herramientas y servicios de apoyo pueden proporcionar al personal educador instrumentos para asegurar la calidad en los programas de doctorado en línea. Pero también lo es que se requiere investigación en este ámbito concreto, y acciones y experiencias en esta vía, que den lugar a modificaciones en el proceso de supervisión entendido como práctica pedagógica.

\section{Metodología}

\section{Propósito}

Se pretende explorar la naturaleza de las relaciones de supervisión y, en particular, los desafíos planteados en el proceso de supervisión a distancia tanto a personal supervisor, como a estudiantes, para adaptarse al nuevo entorno de investigación emergente. En la bibliografía actual, no se encuentra todavía un panorama completo sobre las nuevas prácticas de supervisión, aun cuando se ocupa de algunas expectativas y presenta ejemplos específicos de estas mismas.

El estudio fue diseñado para identificar elementos clave en los procesos de supervisión realizados -mayoritariamente- a distancia, a fin de intervenir y diseñar estrategias de mejora de dichos procesos. Esto requiere averiguar cómo se realiza en la práctica dicha supervisión, cuáles son las destrezas involucradas por parte de las personas supervisoras, las tareas llevadas a cabo y las cualidades de estas que son consideradas factores de éxito. Así, podemos formular como preguntas de investigación:

- ¿Cómo afecta el estilo de supervisión y el apoyo al proceso a la práctica de un doctorado internacional online?

- ¿Cómo es vivido el proceso de supervisión online, tanto desde la perspectiva del personal supervisor como desde la del estudiantado?

- ¿Qué tipo de aplicaciones y herramientas de red son consideradas adecuadas para este tipo de supervisión? 
doi: http://dx.doi.org/10.15359/ree.22-1.4

URL: http://www.una.ac.cr/educare

CORREO: educare@una.cr

Dado que el foco está en identificar elementos donde poder intervenir para mejorar el proceso, se consideró importante contar tanto con la visión de quien supervisa, como de la de investigadores e investigadoras en formación.

Se realizaron entrevistas a estudiantado postgraduado y personal supervisor, con el fin de identificar problemas e interrogantes derivados de las prácticas de supervisión y apoyo. Si pretendemos comprenderlas plenamente, y mejorar la teoría y la práctica de la supervisión de doctorado a distancia, la explicación de lo que significa ser estudiante y un supervisor o una supervisora a distancia resulta una tarea fundamental por realizar.

Cilesiz (2011) propone la fenomenología como enfoque teórico y metodológico para estudiar experiencias con la tecnología como estas. La investigación fenomenológica constituye un intento sistemático de descubrir y describir las estructuras de la experiencia vivida para llegar a una comprensión más profunda de su naturaleza o susignificado (Barnacle 2004; Van Manen, 1990). También hubo preocupación por explorar perspectivas emic del estudiantado y los supervisores y supervisoras captadas a través de sus propias palabras.

El conjunto de actividades de investigación propuestos por Van Manen (1990) es tanto empírico (recogida de la experiencia vivida a través de entrevista conversacional) como reflexivo (reflexión y análisis temático sobre dicha experiencia).

\section{Participantes}

Seseleccionóunamuestraintencionalestratificadadecasos detándem:personasupervisoraestudiante. EL criterio para su selección fue que el proceso de elaboración de la tesis doctoral se hubiera realizado predominantemente online. En primera instancia se seleccionaron las personas supervisoras en relación con la tipología de los estudios de doctorado (convenio específico entre dos instituciones, doctorados internacionales, doctorados nacionales interuniversitarios y doctorado convencionales de una sola universidad). Posteriormente y a partir de los casos que nos proporcionaban dichas personas supervisoras, se seleccionó el estudiantado.

Se identificaron 42 programas de doctorado en ciencias de la educación de universidades españolas susceptibles de supervisar online. Entre ellos, 23 personas supervisoras de 16 instituciones fueron invitadas a participar en las entrevistas, todas ellas en programas de doctorado de educación donde hay matriculado alumnado que investiga en sitios lejanos a la institución: se consiguieron, finalmente, 14 participantes. A cada persona supervisora se le solicito el contacto de uno o dos estudiantes que hubieran completado la investigación y cuya tesis doctoral se hubiera defendido entre 2011 y 2014: se consiguió, al final, entrevistar a 12 estudiantes. Una caracterización más completa puede verse en Tabla 1. 
Tabla 1: Muestra estratificada para las entrevistas

\begin{tabular}{|c|c|c|c|}
\hline Tipo de programa de doctorado & $\begin{array}{l}\text { Supervisor/a } \\
\text { (SUP) }\end{array}$ & $\begin{array}{l}\text { Estudiante } \\
\text { (STU) }\end{array}$ & $\begin{array}{l}\text { TOTAL personas } \\
\text { entrevistadas }\end{array}$ \\
\hline $\begin{array}{l}\text { Convenio específico entre } 2 \text { instituciones (una española y otra } \\
\text { latinoamericana) }\end{array}$ & 7 & 6 & 13 \\
\hline Interuniversitarios internacionales & 1 & 1 & 2 \\
\hline Interuniversitarios nacionales (admiten estudiantes del extranjero) & 3 & 3 & 6 \\
\hline $\begin{array}{l}\text { Convencionales de sola universidad (admiten estudiantes del } \\
\text { extranjero) }\end{array}$ & 3 & 2 & 5 \\
\hline TOTAL & 14 & 12 & 26 \\
\hline
\end{tabular}

Nota: Elaboración propia.

Como se indicó, de la muestra inicial de 23 personas supervisoras se han entrevistado a 14. El resto, o no reunían las condiciones de supervisar online o no había terminado ninguna tesis en el periodo estudiando. En algunos casos no respondieron a la invitación a participar.

Perfil de las personas supervisoras: Profesorado con experiencia en postgrado en la red. Supervisores o supervisores senior en procesos online (entre 5 y 20 años, y entre 3 y 18 casos de tesis doctorales supervisadas parcial o totalmente online, con una media resultante superior a 8 casos de supervisión).

Perfil del estudiantado: Proceden de distintos países latinoamericanos: Venezuela (4), Chile (3); Argentina (1); Brasil (1), México (1), República Dominicana (1), Italia (1). Han terminado su tesis doctoral entre 2011 y 2014 y el proceso de supervisión se ha desarrollado entre 20062014. Son estudiantes de doctorado a tiempo parcial en casi todos los casos (10 de los 12). La mayoría son profesionales y mayoritariamente docentes de universidades, con estancias cortas en la universidad origen del doctorado. La motivación para seguir un programa de doctorado está en la promoción dentro de la carrera de docente (10 de los 12 casos). En 6 casos se trata de algún programa de su propia institución para la promoción. En 7 casos se manifiesta el crecimiento personal o completar la carrera investigadora como motivación.

\section{Recogida y tratamiento de datos}

La recogida de datos se ha realizado mediante entrevistas semiestructuradas, con una primera parte abierta, y una segunda con 5 preguntas cerradas de ordenar o valorar afirmaciones y que fueron realizadas al final a modo de confirmación, conclusión. El propósito del sistema fue implicar a los sujetos participantes en la reflexión, a fin de garantizar que las áreas clave de interés en el estudio fueran abarcadas al mismo tiempo que obtener información valiosa de la espontaneidad de los sujetos entrevistados (Vilkinas, 2008). 
doi: http://dx.doi.org/10.15359/ree.22-1.4

URL: http://www.una.ac.cr/educare

CORREO: educare@una.cr

La entrevista fue seleccionada como método de recogida de información, ya que se presenta como el más apropiado para estudios de carácter exploratorio y son una forma eficiente de recoger datos ricos de la experiencia vivida. Las entrevistas fueron desarrolladas mediante sistemas de videoconferencia (Skype y AdobeConnect), mientras los sujetos entrevistados estaban en sus propios lugares. La entrevista fue abierta y el protocolo de realización de la entrevista incluía las indicaciones necesarias para su desarrollo, atendiendo tanto las formalidades previas como a los contenidos de la entrevista. El audio fue grabado. No se siguió un guion previo, pero quien entrevistaba disponía de una lista de ítems para sugerirlos, en caso de que no surgieran espontáneamente.

Aunque fue tomada en cuenta la revisión de la bibliografía, no se desarrolló un sistema de categorías previo a la realización de las entrevistas, sino que se basan en un primer análisis temático de estas mismas, realizado con anterioridad al análisis línea a línea. Posteriormente, dicho sistema de categorías se aplicó a la transcripción de las entrevistas.

En cuanto a la parte más estructurada de las entrevistas, se presentaron preguntas cerradas relacionadas con las aplicaciones y herramientas, o con un conjunto adicional de proposiciones, que debían ser ordenadas o puntuadas por los sujetos entrevistados. En general, se trata de información ya surgida en la parte más abierta y funcionaba a modo confirmatorio.

\section{Resultados}

Se entiende que es importante conocer el estilo de supervisión y las destrezas implicadas en el caso particular de la supervisión online, así como la forma en que se desarrolla el proceso de supervisión y las herramientas y recursos de comunicación involucrados, todo ello en orden para identificar los aspectos clave involucrados susceptibles de intervención para incorporar ajustes orientados a la mejora del proceso y del producto en la elaboración de las tesis.

\section{Estilo de supervisión y apoyo al proceso}

En relación con el estilo de apoyo y orientación del proceso y la actitud del supervisor o supervisora, la visión de los investigadores o investigadoras en formación varía un poco de la que las personas supervisoras tienen de sí. Para el estudiantado, el personal supervisor proporciona acceso a recursos o la experiencia, la organización de trabajo de campo y domina las técnicas de investigación; y en menor medida muestra interés, discute las ideas del investigador o investigadora en formación. Por su parte, el personal supervisor se ven a sí mismo criticando el diseño de investigación, los borradores de los capítulos, las interpretaciones o datos; dando libertad para tomar decisiones, apoyando las decisiones de cada estudiante; dando ánimo, mostrando interés, discutiendo las ideas, y determinando el tema y forma, aportando ideas. 
Los hallazgos confirman los resultados de Vilkinas (2008) respecto al estilo de supervisión. Las personas supervisoras apoyan intelectualmente, emocionalmente y estructuralmente, y esto lo corroboran los investigadores e investigadoras en formación entrevistados. Hay una gran coincidencia entre personal supervisor y estudiantes en identificar el estilo de supervisión con los atributos de la supervisión activa indirecta según nomenclatura de Gurr (2001), para la alineación del estilo de supervisión desde el momento que es considerado como alguien que pide opiniones y sugerencias, acepta y expande las ideas del estudiantado, y solicita explicaciones y justificaciones de las declaraciones.

En cuanto al proceso de supervisión on-line se solicitó que ante una serie de proposiciones, priorizaran las que requerían mayor apoyo y orientación. Como puede observarse en la Figura 1, el total de los sujetos encuestados (tanto personal supervisor como estudiantes) consideran que se requiere mayor apoyo y orientación en un proceso de supervisión online al construir la estructura de la tesis (a); organizar un buen plan de trabajo y seguimiento (g), y organizar y conectar la investigación con conceptos y relaciones del dominio de conocimiento donde se investiga (c). Si atendemos las proposiciones con mayor frecuencia en el primer lugar, las dos primeras coinciden.

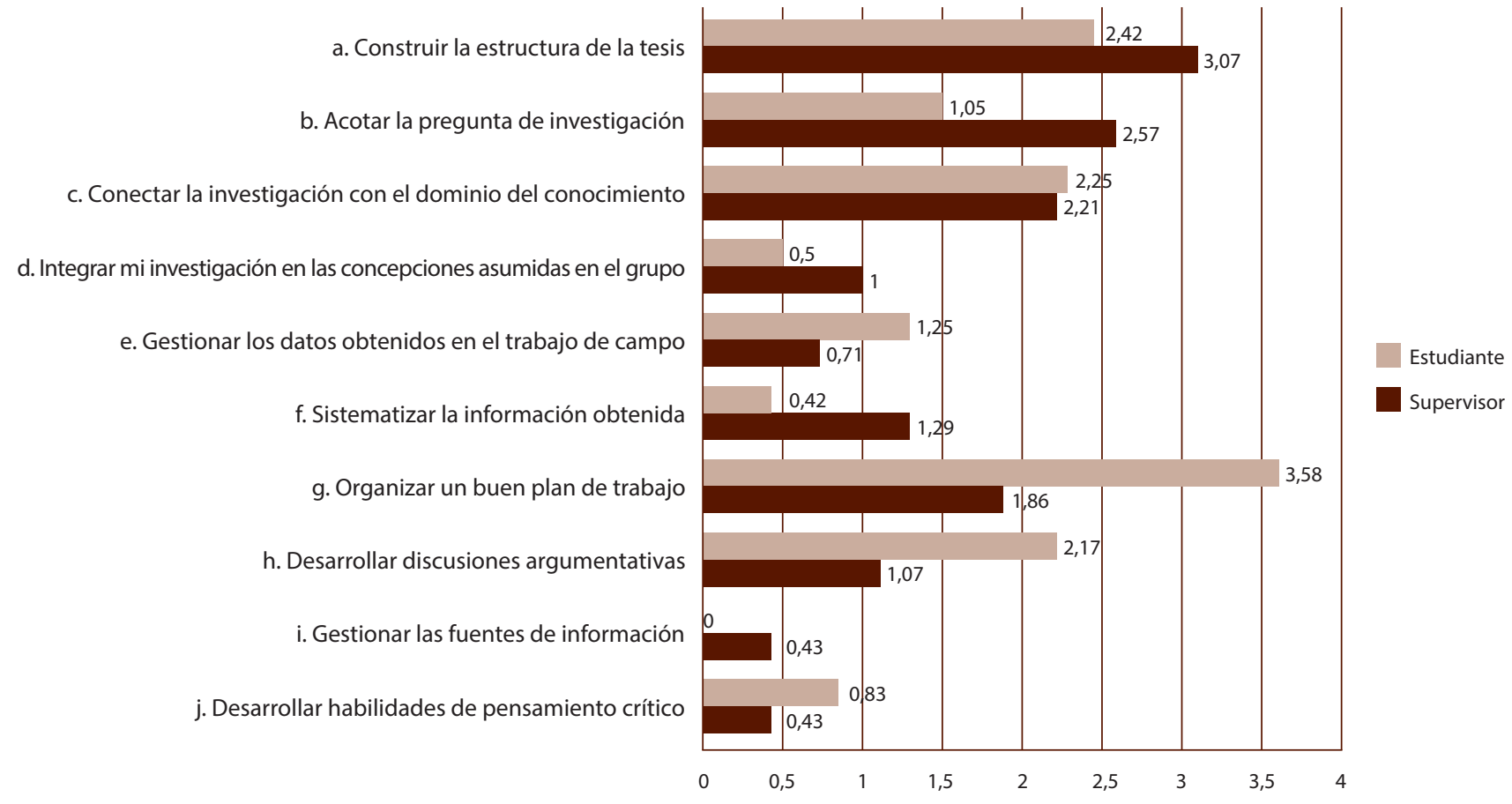

Figura 1: Acciones que requieren mayor apoyo y orientación. 
doi: http://dx.doi.org/10.15359/ree.22-1.4

URL: http://www.una.ac.cr/educare

CORREO: educare@una.cr

Hay algunos ítems en los que se ha encontrado diferencias significativas entre ambos grupos. Por una parte, investigadores e investigadoras en formación dan mayor importancia que los supervisores y supervisoras a organizar un buen plan de trabajoy seguimiento (g) y desarrollar discusiones argumentativas y explicitar la argumentación (h). Por el contrario, encontramos grandes diferencias entre ambos colectivos en la importancia que dan a la construcción de la estructura de doctorado (a) y sobre todo en acotar la pregunta de investigación (b). Si bien todas estas características son parte esencial de la supervisión en línea, la oferta y la demanda parecen necesitar un punto de encuentro para un cambio en el estilo de supervisión y mejorar los modos de comunicación.

Una cuestión interesante hace referencia a las diferencias culturales. Los comentarios surgen principalmente entre personal supervisor $y$, con algunas reservas, podría ser analizado desde la perspectiva de la teoría postcolonial tal como Manathunga (2009) y Grant (2010) la aplican a la supervisión intercultural. A modo de ejemplo: “Hay problemas de comunicación, probablemente debido a las diferencias culturales" (SUP3); "Aparecen brechas de comunicación que no se pueden atribuir a la comunicación online" (SUP6); "Tienen otra organización mental ..." (SUP9); “La investigación se realiza en el contexto del estudiante ..." (SUP10); "Mismo idioma no significa misma cultura ..." (SUP12); "Tienes que ponerte en el contexto del estudiante ..." (SUP8). Estas diferencias culturales son vistas entre el mundo del personal supervisor y el del "otro", sin tener en cuenta, por ejemplo, las diferencias entre los distintos países de origen de los investigadores e investigadoras en formación o el bagaje cultural que pueden traer.

En general, ambos grupos ven positivo el proceso online. La relación personal supervisor/ estudiantado supervisado es descrita como una evolución hacia una relación entre colegas en lugar de una relación jerárquica y, a veces, la colaboración en la investigación continúa después de la finalización del doctorado.

\section{Dinámica del proceso}

Uno de los principales propósitos de este trabajo es explorar los desafíos del proceso de supervisión online tanto desde la visión del personal supervisor, como de la del estudiantado. Para ello, lo primero por averiguar es cómo se realiza en la práctica dicha supervisión, cuales son las destrezas comunicativas involucradas por parte de supervisores y supervisoras, las tareas llevadas a cabo y las herramientas en las que se apoyan.

Las principales ventajas de trabajar en línea durante el proceso de elaboración de la tesis doctoral manifestadas por el personal supervisor hacen referencia a la flexibilidad y a la precisión de la comunicación escrita y el trabajo sobre documentos. Para el estudiantado, aunque señala también estas mismas ventajas, la más señalada (8/12 casos) es la disponibilidad 
del supervisor o supervisora. En este punto conviene recordar que la mayoría de investigadores e investigadoras en formación entrevistada son profesionales en ejercicio y mayoritariamente docentes de universidades, cuya motivación para realizar un programa de doctorado está en la promoción dentro de la carrera de profesorado.

Paradójicamente, la comunicación escrita es vista al mismo tiempo como uno de los inconvenientes más importantes por el esfuerzo que supone las sucesivas lecturas y la anotación sobre documentos, esto es señalado tanto por personal supervisor como por estudiantes. Según la supervisión, los problemas de compresión y de consenso de significados son los más graves (10/14 casos), mientras en el estudiantado es el aislamiento (8/12 casos).

En relación con el proceso de comunicación entre personal supervisor y estudiante, se ha encontrado que en todos los casos el medio de comunicación principal ha sido el correo electrónico, con intercambio de documentos adjuntos. Sobre estos, ha sido habitual incorporar anotaciones a los documentos con observaciones, comentarios, etc., aunque hay algún caso en los que estos se devuelven en un documento aparte.

12 de las 14 personas que realizan la supervisión utilizan como herramienta alternativa la herramienta Skype, preferentemente chat o combinado con videoconferencia. Solamente 2 personas supervisoras manifiestan haber utilizado un sistema de documentos compartidos concretamente Google-docs.

En cuanto a cómo podría mejorarse el proceso de comunicación en la supervisión, pueden agruparse las respuestas en dos grupos:

- Mejoras dirigidas al proceso mismo. Comentarios en este sentido:"Para mí es importante tener un horario de trabajo acordado con el supervisor" (STU2); "Propongo un programa de reuniones formales y el compromiso por parte del estudiante" (STU6); “Es necesario que haya compromiso de ambas partes" (SUP3); “Un contrato personal entre ellos mejoraría esto" (SUP8). En este caso, la conveniencia de tener un cronograma de trabajo conjunto o un contrato es mencionado en repetidas ocasiones. En este sentido, el estudiantado considera positivo tener un horario de trabajo y un compromiso.

- Mejoras dirigidas al sistema de comunicación. Comentarios en este sentido:“Debemos proporcionar recursos para la investigación .... Deseable contar con herramientas que faciliten la supervisión, el diálogo sobre las observaciones..." (SUP1); “Es necesario tener un espacio permanente para organizar documentos específicos y comunicar" (SUP2); "Me gustaría proponer un sistema de gestión del aprendizaje especial para el doctorado donde incorporar la comunicación interpersonal" (STU3); "Puede ser positivo generar una comunidad de estudiantes" (SUP6); "Echo de menos herramientas de trabajo colaborativo (para colaborar en la escritura)" (SUP4); "Videoconferencia, documentos 
doi: http://dx.doi.org/10.15359/ree.22-1.4

URL: http://www.una.ac.cr/educare

CORREO: educare@una.cr

compartidos y un sistema para ver automáticamente el progreso" (SUP11). Con respecto a las ventajas, se menciona el uso de un sistema de documentos compartidos (Google-docs, Titanpad, Co-ment, etc.). En el caso de una parte de estudiantes, este uso se considera de interés específico para el programa de doctorado. El uso más frecuente de la videoconferencia es mencionado por la totalidad de participantes, tanto por parte de supervisores y supervisoras, como de investigadores e investigadoras en formación.

\section{Aplicaciones y herramientas de red}

Como aspectos específicos de los procesos de supervisión online, es importante atender el uso y la necesidad de dominar distintos tipos de herramientas software disponibles que facilitan realizar funciones de gestión del conocimiento en entornos virtuales relacionados con el proceso de supervisión y con el proceso completo de realización de la investigación.

Se propusieron seis grupos de herramientas en relación con dichas funciones para que se priorizaran. Cuando se pide que se considere cuáles son conveniente que sean dominadas y utilizadas por el investigador o investigadora en formación, en todos los casos aparecen en primer lugar las herramientas asociadas a 'buscar, clasificar y filtrar', tal como puede verse en la Figura 2, pero mientras que para los supervisores y supervisoras son puntuadas a continuación "herramientas para personalizar y organizar" y'almacenar y recuperar'; para el estudiantado son las herramientas de 'gestión de información' y 'crear y representar' las que siguen.

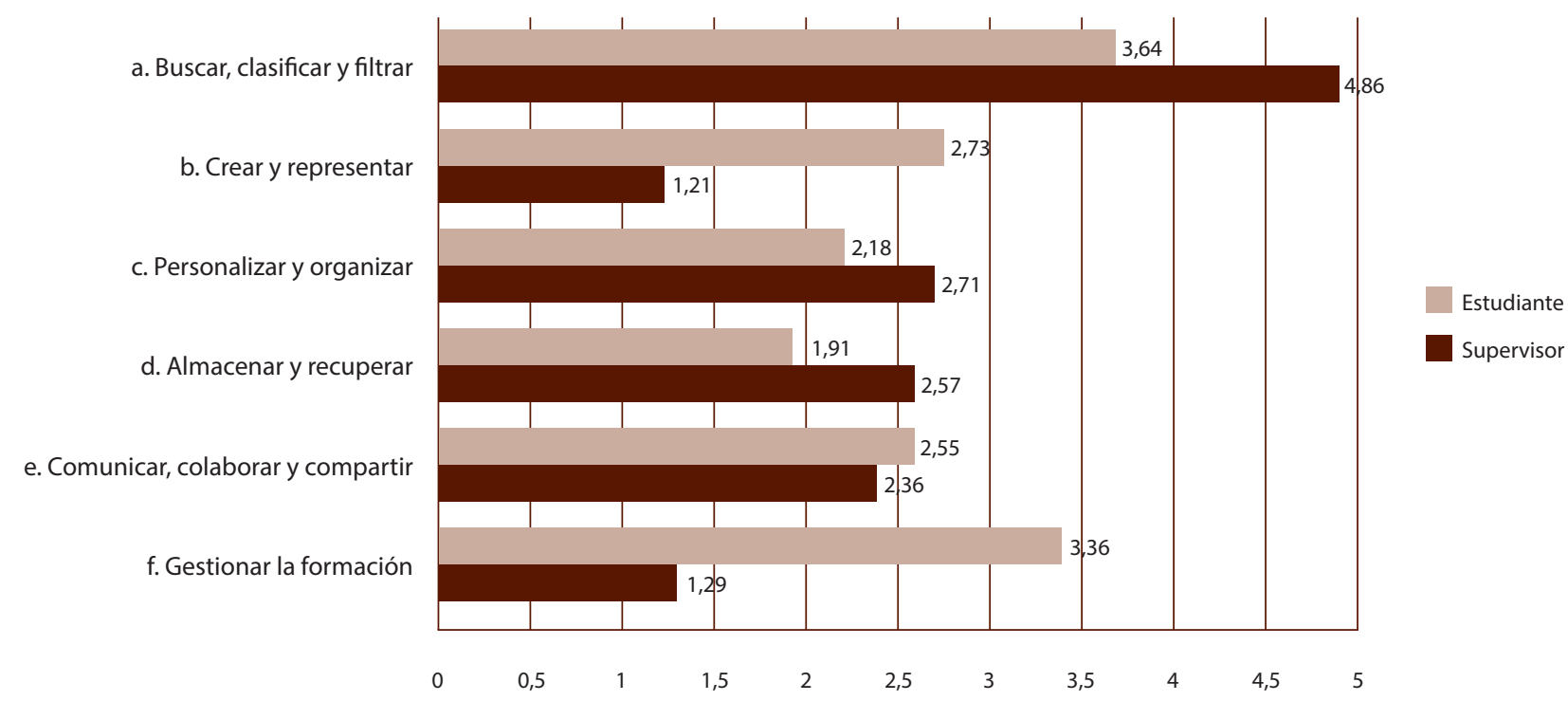

Figura 2: Herramientas que deben ser dominadas y usadas por el estudiantado. 
Cuando la cuestión es acerca de las herramientas que debería dominar y utilizar quien supervisa, también hay coincidencia entre supervisores y supervisoras e investigadores e investigadoras en formación en considerar, como las más valoradas, las herramientas de 'comunicación, colaborar y compartir' (véase Figura 3). En este caso, supervisores y supervisoras puntúan en segundo lugar las de 'buscar, clasificar y filtrar', mientras para el estudiantado son las herramientas de 'gestión de la información' las que también deben dominar y utilizar los sujetos supervisores.

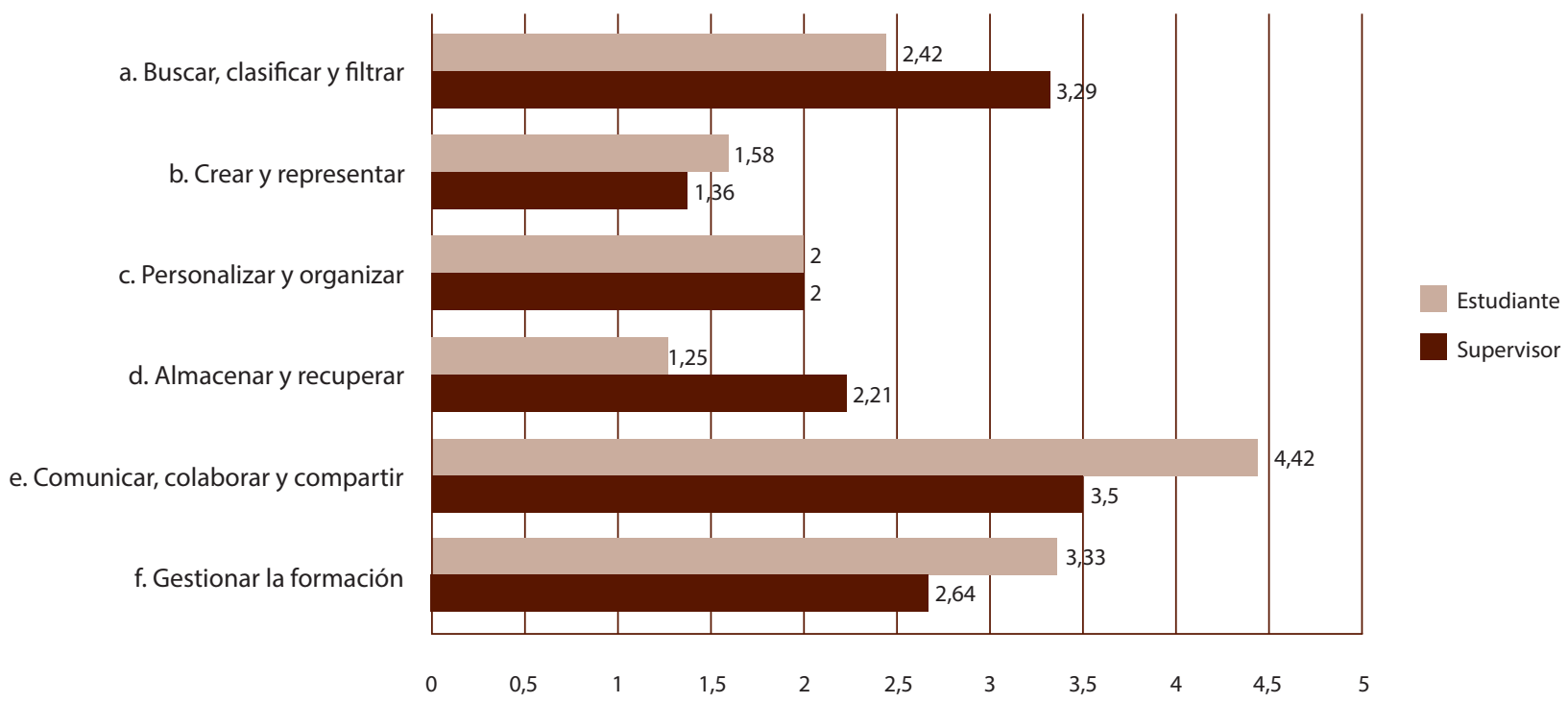

Figura 3: Herramientas que deben ser dominadas y usadas por el supervisor.

Cuando se solicita que se valore cómo la utilización de cada uno de los seis grupos de herramientas mejoraría el proceso y el producto de la supervisión, siempre en relación con las funciones de gestión del conocimiento en entornos virtuales, aparecen ciertas discrepancias entre supervisores y supervisoras e investigadores e investigadoras en formación. Los sujetos supervisores valoran como herramientas que contribuirían a mejorar el proceso las de 'buscar, clasificar y filtrar' junto a 'comunicación, colaborar y compartir'; mientras que el estudiantado valora más las de 'personalizar y organizar' y'almacenar y recuperar' (Figura 4). 
doi: http://dx.doi.org/10.15359/ree.22-1.4

URL: http://www.una.ac.cr/educare

CORREO: educare@una.cr

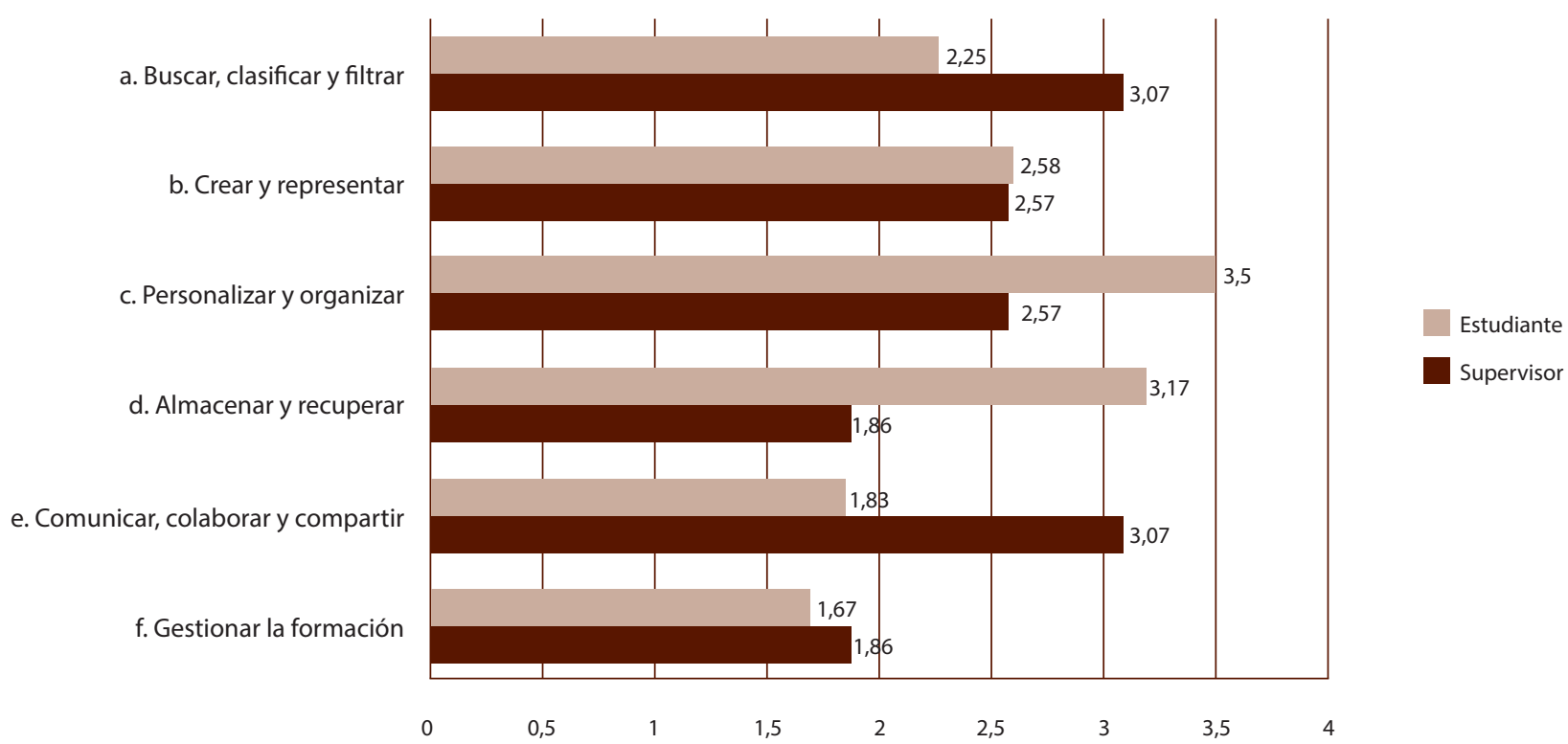

Figura 4: Herramientas que mejoran el proceso y la productividad de la supervisión.

En cuanto a qué tareas del proceso de las descritas en la Figura 1 pueden ser reforzadas con el uso de aplicaciones y herramientas en red, encontramos grandes diferencias entre ambos grupos (Figura 5). Entre supervisores y supervisoras, los ítems más valorados fueron: gestionar los datos e información obtenidos en el trabajo de campo (e), gestionar las fuentes de información (i) y construir la estructura de la tesis (a). Entre el estudiantado, por el contrario, los más valorados fueron: sistematizar la información obtenida (f), organizar un buen plan de trabajo y seguimiento ( $\mathrm{g}$ ), y gestionar los datos e información obtenidos en el trabajo de campo (e). Excepto para el ítem (e), encontramos grandes diferencias en la valoración de cada grupo.

El análisis sugiere que la supervisión de la calidad significa conocer las mejores prácticas en el uso del e-learning, llevar a cabo un sistema de gestión de aprendizaje y experiencia en la gestión de la información personal. 


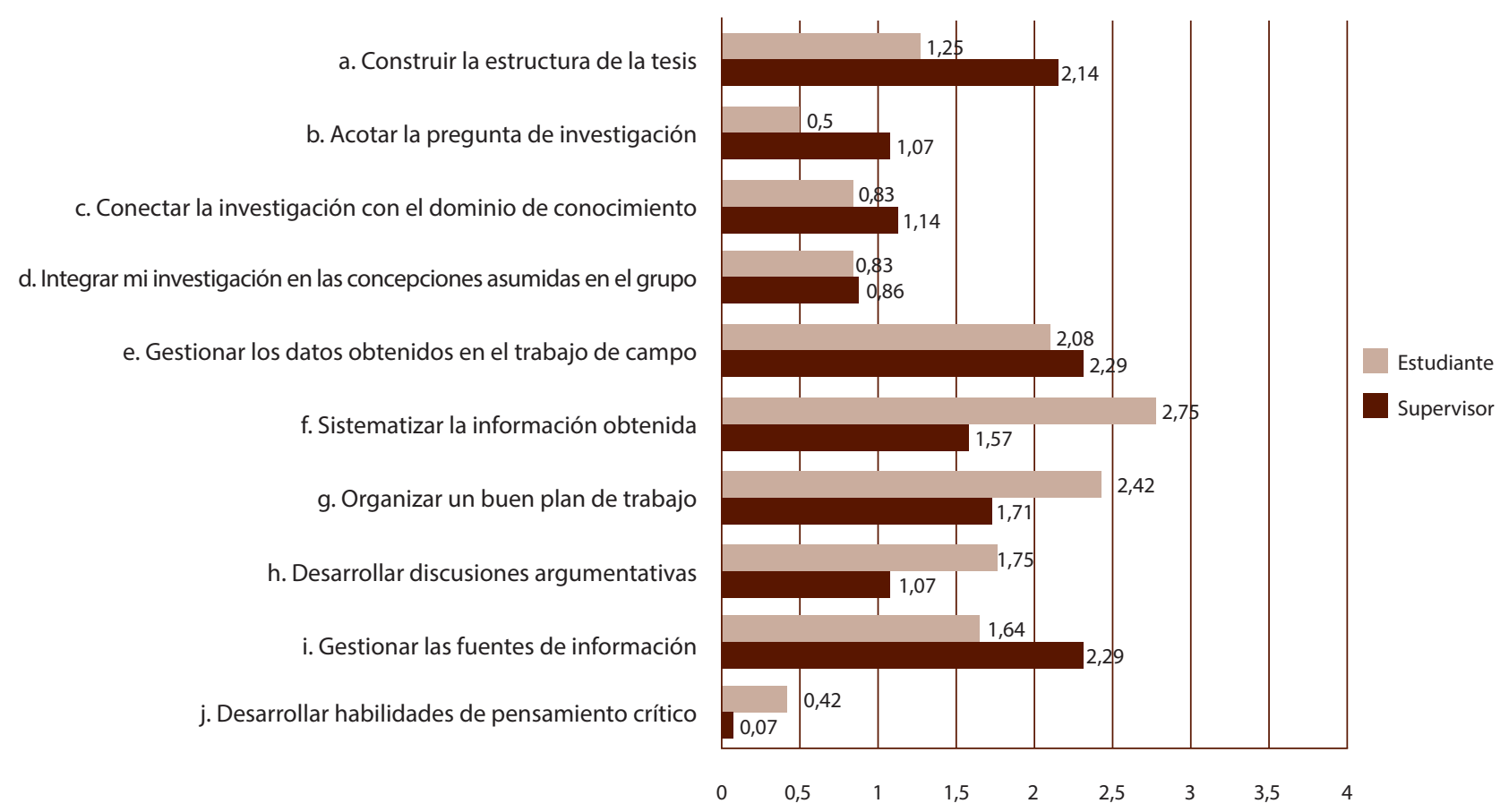

Figura 5: Acciones que pueden mejorarse con el uso de aplicaciones y herramientas de red.

\section{Conclusión}

Antes que nada, conviene matizar las conclusiones tanto en función del ámbito geográfico del estudio, como por el campo de estudio de los doctorados. Respecto al ámbito geográfico, hay que señalar que, al tratarse de doctorados en español con una extracción de estudiantado latinoamericano, algunas de las reflexiones deben circunscribirse a este ámbito concreto. Respecto al campo de conocimiento, cabe señalar que las ciencias de la educación presentan una casuística peculiar respecto a los procesos de supervisión online, como son realizar las investigaciones de campo fuera del laboratorio $y$, en general, fuera de la universidad; personal supervisor sensible a priori hacia las propuestas pedagógicas respecto al doctorado, entre otras, tal como señalan Erichsen et al. (2014) e Ives y Rowley (2005).

Por otra parte, vemos a posteriori como un acierto el haber entrevistado a las dos partes del proceso, ya que nos permitió contrastar y relacionar la experiencia vivida por ambos colectivos. Dado que la atención se centra en identificar los elementos en los que intervenir para mejorar el proceso, se consideró importante tener en cuenta tanto los puntos de vista de supervisores y supervisoras como de investigadores e investigadoras en formación. Existe coincidencia en señalar los elementos clave en relación con el estilo de supervisión y apoyo al proceso, a la dinámica 
doi: http://dx.doi.org/10.15359/ree.22-1.4

URL: http://www.una.ac.cr/educare

CORREO: educare@una.cr

o en relación a las herramientas y aplicaciones susceptibles de mejorar el proceso, pero ambos colectivos no le atribuyen el mismo valor, tal como se muestró en los resultados correspondientes.

El objetivo del estudio era identificar los elementos clave en los procesos de supervisión realizados -mayoritariamente- a distancia, en unos casos concretos con las características ya señaladas. Esto requiere averiguar cómo se ha vivido la experiencia; cómo se realiza en la práctica dicha supervisión; cuáles son las destrezas involucradas por parte del personal supervisor, las tareas llevadas a cabo y las características de este que son consideradas factores de éxito. Todo ello a fin de identificar los elementos más débiles donde poder intervenir para mejorar tanto el proceso como el entorno de comunicación donde se desarrolla.

Algunas de las características de la buena supervisión asumidas en la revisión de la bibliografía toman especial importancia en la supervisión online:

- Lo primero a destacar es que tanto personal supervisor como estudiantado supervisado se manifiestan satisfechos con el proceso, si bien señalan las limitaciones de este. Así mismo, cabe destacar la satisfacción del estudiantado con la labor de supervisión y con la actitud en la relación entre ambas partes, que aun siendo percibida como desigual, ha perdido gran parte de la connotación jerárquica. En nuestro caso, la mayoría del estudiantado proviene del personal académico de instituciones de educación superior. Como señala Denicolo (2004, citado por Watts 2010), la relación entre quien supervisa y la persona supervisada podría ser vista como entre pares, lo que requiere la adaptación del comportamiento por ambas partes. Esto coincide también con los hallazgos de Malfroy (2005).

- Serequiereadaptacióncultural porambaspartes.Semencionan las dificultades causadas por las diferencias culturales, por lo que sería interesante mayor investigación a la luz de la teoría postcolonial, así como la aplicación de conceptos como transculturación o zona de contacto (Grant 2010; Manathunga 2009), donde la diferencia cultural es percibida como productiva y no como una desventaja. Así, participar en la investigación en la zona de contacto intercultural permite a quienes investigan aprovechar las sinergias, las interconexiones y las relaciones para desarrollar nuevos conocimientos y formas de llevar a cabo la investigación.

- Los procedimientos de seguimiento online no cambian mucho por parte del personal supervisor, excepto en el sistema de comunicación utilizado, pero el estudiantado se encuentra con grandes cambios debido a que el doctorado es a menudo seguido a tiempo parcial en una institución con una cultura extraña. Cuando el estudiantado valora la disponibilidad del personal supervisor, no solo está hablando de aspectos de la comunicación; sino que también se refiere a una actitud hacia perspectivas culturales. 
- Los aspectos del proceso de supervisión on-line que requieren un mayor grado de apoyo y orientación por los sujetos entrevistados, tomados globalmente, son por orden de valoración: Construir la estructura de la tesis, organizar un buen plan de trabajo y seguimiento, y organizar y conectar la investigación con conceptos y relaciones del dominio de conocimiento donde se investiga. Por separado, personal supervisor y estudiantado supervisado coinciden con ello, aunque no en el mismo orden ni con igual importancia (por ejemplo, hay una fuerte diferencia entre estudiantes y personal supervisor en relación con la importancia de la necesidad de "organizar un buen plan de trabajo").

- Respecto a las principales ventajas de la supervisión online, los supervisores y las supervisoras hacen referencia a la flexibilidad del proceso y a la precisión de la comunicación escrita, aunque para el estudiantado, la más señalada es la disponibilidad del personal supervisor.

- En cuanto a las dificultades en el proceso, entre supervisores y supervisoras, los problemas de compresión y de consenso de significados son los más graves, mientras en el estudiantado es el aislamiento. Aspectos de la comunicación escrita son vistos al mismo tiempo, por ambos grupos, como inconvenientes por el esfuerzo que supone las sucesivas lecturas y la anotación sobre documentos.

Existen evidencias que sugieren que la calidad de la supervisión online significa conocer buenas prácticas en el uso del aprendizaje conectado, disponer de un sistema de gestión del aprendizaje y ser capaz de orientar a los sujetos candidatos en el uso de las herramientas de gestión de la información personal. Al respecto, Strang (2009) señala:

- Se constata la necesidad de un mayor manejo de las herramientas existentes de gestión del conocimiento en entornos virtuales para mejorar el proceso de supervisión y el proceso completo de realización de la investigación.

- Los procedimientos de supervisión utilizados en los casos estudiados se apoyan en unas pocas herramientas básicas, donde el correo electrónico todavía dispone de un espacio consolidado. El chat y la videoconferencia también son utilizados. Desde la visión de las herramientas de red disponibles en la actualidad, podría definirse como un procedimiento rudimentario para los procesos comunicativos que se ven involucrados en la elaboración de una tesis.

- Una vía para lograrlo parece estar en liberar a las personas supervisoras de la dependencia de la comunicación escrita como sistema y objeto del trabajo de supervisión, para pasar a lograr una mejor comunicación oral sobre distintos recursos de representación del conocimiento (esquemas, líneas de tiempo, mapas conceptuales, 
doi: http://dx.doi.org/10.15359/ree.22-1.4

URL: http://www.una.ac.cr/educare

CORREO: educare@una.cr

diagramas), sobre todo en las fases iniciales y intermedias del proceso (construcción de la estructura de la tesis, conexión con la teoría y el marco de referencia, elaboración de las preguntas de investigación, etc.).

- También parece importante disponer de algún tipo de sistema para organizar y compartir recursos de investigación que podría ser específico del programa de doctorado. Un ejemplo de ello podría ser un entorno virtual de aprendizaje específico o una comunidad virtual de investigadores e investigadoras en formación.

Puede parecer contradictorio reducir el análisis de la relación personal supervisorestudiante a una en la que este último grupo es considerado un novato que se embarca en el difícil proceso de adquisición de conocimientos complejos, un proceso orquestado dirigido por el conocimiento del supervisor o supervisora, sin tener en cuenta las habilidades que el investigador o investigadora en formación aporta al proyecto, así como sus intereses, las diferencias culturales y las necesidades. Y esto se hace más importante para la supervisión online, tanto por la sensación de aislamiento del alumnado, como porque esos intereses, diferencias culturales, y necesidades son más difíciles de demostrar al supervisor como un resultado. Esto es fundamental para fortalecer el entorno personal de aprendizaje para el estudiantado. El dominio de las herramientas para la gestión de información personal orientada a la investigación por ambas partes mejorará el proceso de supervisión online.

\section{Referencias}

Barnacle, R. (2004). Reflection on lived experience in educational research. Educational Philosophy and Theory, 36(1), 57-67. doi: https://doi.org/10.1111/j.1469-5812.2004.00048.x

Cilesiz, S. (2011). A phenomenological approach to experience with technology: Current state, promise, and future directions for research. Educational Technology Research and Development, 59(4), 487-510. doi: https://doi.org/10.1007/s11423-010-9173-2

Combe, C. (2005). Developing and implementing an online doctoral programme. International Journal of Educational Management, 19(2), 118-127. doi: https://doi. org/10.1108/09513540510582417

Edwards, B. (December, 2002). Postgraduate supervision: Is having a Ph.D. enough? Paper presented at the Australian Association for Research in Education Conference "Problematic futures: Educational research in a era of uncertainty. Brisbane, Australia. Retrieved from https://www.aare.edu.au/data/publications/2002/edw02382.pdf

Erichsen, E. A., Bolliger, D., U., \& Halupa, C. (2014). Student satisfaction with graduate supervision in doctoral programs primarily delivered in distance education settings. Studies in Higher Education, 39(2), 1-18. doi: https://doi.org/10.1080/03075079.2012.709496 
European University Association (EUA). (2007). Doctoral programmes in europe's universities: Achievements and challenges. Report prepared for european universities and ministers of higher education. Belgium: Autor. Retrieved from https://www.dcu.ie/sites/default/files/ graduate research/pdfs/doctoral programmes in europe $s$ universities.pdf

Evans, T. (2010). Supervising part-time doctoral students. In M. Walker, \& P. Thomson (Eds.), The routledge doctoral supervisor's companion. Support effective research in education and the social sciences (pp. 131-137). London and New York: Routledge.

Grant, B. M. (2010). Challenging matters: Doctoral supervision in post-colonial sites. Acta Academica Supplementum, 1, 103-129. Retrieved from http://scholar.ufs.ac.za:8080/xmlui/ bitstream/handle/11660/2699/academ supp1 2010 a5.pdf?sequence=1\&isAllowed=y

Green, B. (2009). Challenging perspectives, changing practices: Doctoral education in transition. In D. Boud, \& A. Lee (Eds.), Changing practices of doctoral education (pp. 239-248). London and New York: Routledge.

Gilbert, R. (2004). A framework for evaluating the doctoral curriculum. Assessment \& Evaluation in Higher Education, 29(3), 299-309. doi: https://doi.org/10.1080/0260293042000188258

Golde, C. M. (2005). The role of the department and discipline in doctoral student attrition: Lessons from four departments. The Journal of Higher Education, 76(6), 669-700. doi: https://doi.org/10.1080/00221546.2005.11772304

Gurr, G. M. (2001). Negotiating the "Rackety Bridge" — a Dynamic model for aligning supervisory style with research student development. Higher Education Research \& Development, 20(1), 81-92. doi: https://doi.org/10.1080/07924360120043882

Hartikainen H., Suhonen J., \&Sutinen E. (2006).IMPDET-anOnlinePhDstudyprogram ineducational technology. In Proceeding ICALT 06 Proceedings of the sixth IEEE International Conference on Advanced Learning Technologies (578-580). Finland: Department of Computer Science, University of Joensuu. doi: https://doi.org/10.1109/ICALT.2006.1652508

Ives, G., \& Rowley, G. (2005). Supervisor selection or allocation and continuity of supervision: Ph. D. students' progress and outcomes. Studies in Higher Education, 30(5), 535-555. doi: https://doi.org/10.1080/03075070500249161

Latona, K., \& Browne, M. (2001). Factors associated with completion of research higher degrees. Canberra, Australia: Higher Education Division Department of Education, Training and Youth Affairs.

Lee, A. (2008). How are doctoral students supervised? Concepts of doctoral research supervision. Studies in Higher Education, 33(3), 267-281. doi: https://doi.org/10.1080/03075070802049202 
doi: http://dx.doi.org/10.15359/ree.22-1.4

URL: http://www.una.ac.cr/educare

CORREO: educare@una.cr

Lee, A., \& Boud, D. (2009). Framing doctoral education as practice. In D. Boud, \& A. Lee, (Eds.), Changing Practices of Doctoral Education (pp. 10-25). London and New York: Routledge.

Lessing, A.C., \& Schulze, S. (2003). Lecturers' experience of postgraduate supervision in a distance education context. South African Journal of Higher Education, 17(2), 159-168. Retrieved from http://uir.unisa.ac.za/bitstream/handle/10500/199/ar schulze lecturerexperience. pdf? sequence $=1$ \&isAllowed $=y$

Macauley, P. (2002). Doctoral research at a distance: Are the deficits illusory? In T. D. Evans, (Ed.), Research in Distance Education: revised papers from the fifth Research in Distance Education Conference, (pp. 64-76). Geelong, Victoria: Deakin University.

Malfroy, J. (2005). Doctoral supervision, workplace research and changing pedagogic practices. Higher Education Research \& Development, 24(2), 165-178. doi: https://doi. org/10.1080/07294360500062961

Manathunga, C. (2009). Research as an intercultural'contact zone'. Discourse: Studies in the Cultural Politics of Education, 30(2), 165-177. doi: https://doi.org/10.1080/01596300902809161

Orellana, M. L., Darder, A., Pérez, A., \& Salinas, J. (2016). Improving doctoral success by matching PhD students with supervisors. International Journal of Doctoral Studies (IJDS), 11, 87-103. Recuperado de http://ijds.org/Volume11/IJDSv11p087-1030rellana1629.pdf

Price, D. C., \& Money, A. H. (2002). Alternative models for doctoral mentor organisation and research supervision. Mentoring \& Tutoring: Partnership in Learning, 10(2), 127-135. doi: https://doi.org/10.1080/1361126022000002446

Seagram, B. C., Gould, J., \& Pyke, S. W. (1998). An investigation of gender and other variables on time to completion of doctoral degrees. Research in Higher Education, 39(3), 319-35. Retrieved from https://doi.org/10.1023/A:1018781118312

Sinclair, M. (2004). The pedagogy of 'good' PhD supervision: A national cross-disciplinary investigation of PhD supervision. Canberra: Faculty of Education and Creative Arts, Central Queensland University. Retrieved from http://site.uit.no/epinor/files/2013/08/ThePedagogy-of-Good-PhD-Supervision.pdf

Strang, K. D. (2009). Measuring online learning approach and mentoring preferentes of international doctorate students. International Journal of Educational Research, 48(4), 245257. doi: https://doi.org/10.1016/j.jjer.2009.11.002

Unwin T. (2007). Reflections on supervising distance-based PhD students. Retrieved from http:// www.gg.rhul.ac.uk/ict4d/distance-based\%20PhDs.pdf 
Watts, J. H. (2010). Supervising part-time doctoral students. Issues and challenges. In M. Walker, \& P. Thomson (Eds.), The routledge doctoral supervisor's companion. Support effective research in education and the social sciences (pp. 123-130). London and New York: Routledge.

Van Manen, M. (1990). Researching lived experience. Human science for an Action sensitive pedagogy. Albany, NY: State University of New York Press.

Vilkinas, T. (2008). An exploratory study of the supervision of Ph.D./Research students' theses. Innovative Higher Education, 32(5), 297-311. doi: https://doi.org/10.1007/s10755-0079057-5

Villardón-Gallego, L. y Yániz, C. (2013). Propuesta de un plan de tutoría y apoyo a estudiantes de doctorado. REDU Revista de Docencia Universitaria, 11(2), 135-152. Recuperado de https:// polipapers.upv.es/index.php/REDU/article/view/5570/5560

Wikeley, F., \& Muschamp. Y. (2004). Pedagogical implications of working with doctoral students at a distance. Distance Education, 25(1), 125-142. doi: https://doi. org/10.1080/0158791042000212495

Winston, B. E., \& Fields, D. L. (2003). Developing dissertation skills of doctoral students in an internet-based education curriculum: a case study. American Journal of Distance Education, 17(3), 161-172. doi: https://doi.org/10.1207/S15389286AJDE1703 3

Woolderink, M., Putnik, K., van der Boom, H., \& Klabbers, G. (2015). The voice of PhD candidates and $\mathrm{PhD}$ supervisors. A qualitative exploratory study amongst $\mathrm{PhD}$ candidates and supervisors to evaluate the relational aspects of PhD supervision in the Netherlands. International Journal of Doctoral Studies, 10, 217-235. Recuperado de http://ijds.org/ Volume10/IJDSv10p217-235Woolderink0852.pdf 